\title{
The Impact of Pedestrian and Nonmotorized Vehicle Violations on Vehicle Emissions at Signalized Intersections in the Real World: A Case Study in Beijing
}

\author{
Jianchang Huang $\mathbb{D}$, ${ }^{1}$ Guohua Song $\mathbb{D},{ }^{1}$ Jianbo Zhang $\mathbb{D}^{2},{ }^{2}$ Zufen Li $\mathbb{D},{ }^{1}$ Yizheng Wu $\mathbb{D}$, \\ and Lei $Y u \mathbb{D}^{1,3,4}$ \\ ${ }^{1}$ Key Laboratory of Transport Industry of Big Data Application Technologies for Comprehensive Transport, \\ Beijing Jiaotong University, Beijing 100044, China \\ ${ }^{2}$ Department of Intelligent Transportation System, Beijing Transport Institute, Beijing 100073, China \\ ${ }^{3}$ Department of Transportation Studies, Xuchang University, Xuchang 461000, China \\ ${ }^{4}$ Department of Transportation Studies, Texas Southern University, 3100 Cleburne Avenue, Houston, TX 77004, USA
}

Correspondence should be addressed to Guohua Song; ghsong@bjtu.edu.cn

Received 29 May 2020; Revised 8 July 2020; Accepted 6 February 2021; Published 2 March 2021

Academic Editor: Keping Li

Copyright $\odot 2021$ Jianchang Huang et al. This is an open access article distributed under the Creative Commons Attribution License, which permits unrestricted use, distribution, and reproduction in any medium, provided the original work is properly cited.

\begin{abstract}
Emission around intersections has become an issue in the urban traffic network. This paper aims to investigate the impact of pedestrian and nonmotorized vehicle violations on emissions at mixed-traffic flow intersection based on the volumes of vehicles, nonmotor vehicles, and pedestrians. Also, it focuses on the arterial and collector intersections with high vehicle volume and limited space. Running red light and crossing intersection diagonally are two critical violations, accounting for $91.75 \%$ of effective violations (interference with vehicles' operation). In this context, a violation blocking model is developed to estimate the blocking probability for each vehicle based on the volumes of pedestrians and nonmotor vehicles. The model includes two scenarios. (1) Through phase: the violation blocking model of running red light is developed based on the survival curve (the relationship between waiting time and running red light probability). (2) Left-turn phase: the violation blocking model at this phase includes two parts: (i) crossing the intersection diagonally model is developed for the first vehicle and (ii) running red light model is developed for subsequent vehicles. The existing emission model can estimate the emissions based on the blocking positions. In the case study, emissions increase with the vehicle volume approaching the saturated flow rate and the volumes of nonmotor vehicles and pedestrians increasing. Results show that the maximum emission increase of $\mathrm{CO}$ (carbon monoxide) for through phase and left-turn phase can reach $16.7 \%$ and $36.4 \%$.
\end{abstract}

\section{Introduction}

Emission around intersections has become an issue in the urban traffic network. [1], especially in densely populated metropolitans. Previous studies illustrate that high emissions mainly resulted from the stop-and-go vehicle activities at the intersection [2-4], which further lead to high pollutant exposure pedestrians around the intersections [5].

Violation behaviors frequently exist in developing countries such as China [6]. Frequent violations can not only merely intensify the traffic risk but also increase the stop- and-go activities (see Figure 1). Few studies have investigated the impact of violations of pedestrians and nonmotorized vehicles on the vehicle emissions at real world intersections. A violation blocking model based on real world data is recently developed instead of hypothetical violations [7]. In this paper, the main problem is that how many emissions were caused by pedestrian and nonmotorized vehicle violations based on the volumes of vehicles, nonvehicles, and pedestrians in the real world.

A lot of research focused on intersection emissions, which could be summarized into six categories: the influence 


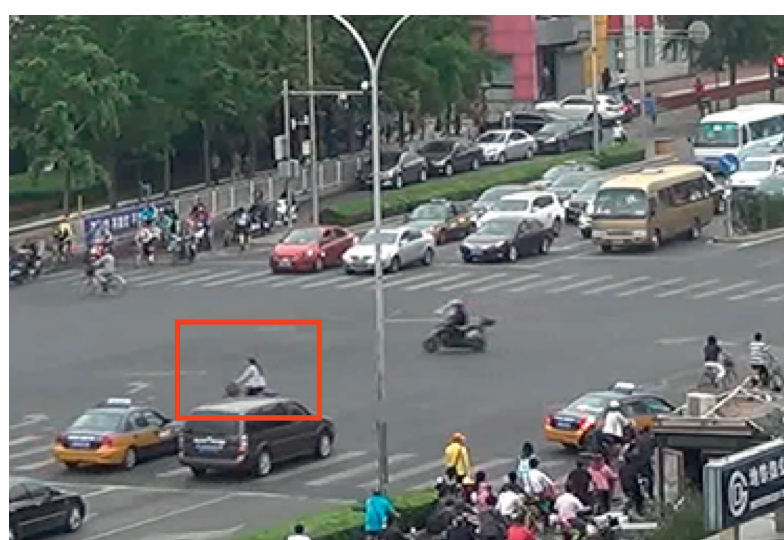

(a)

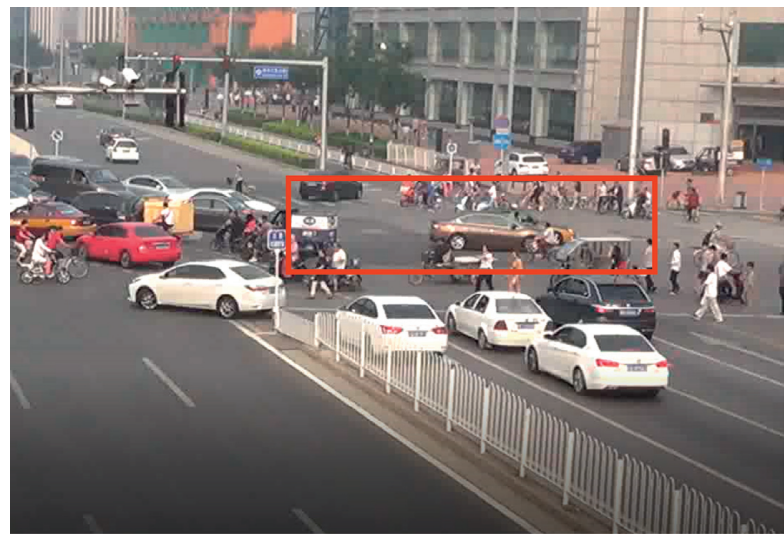

(c)

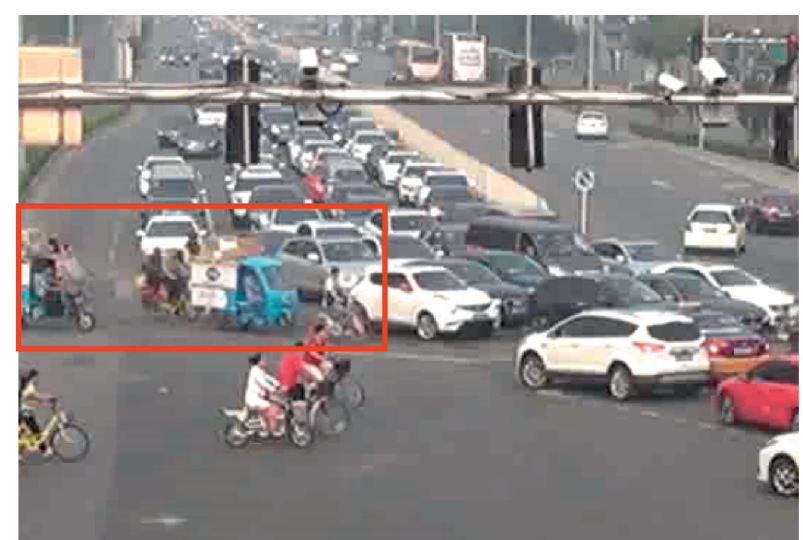

(b)

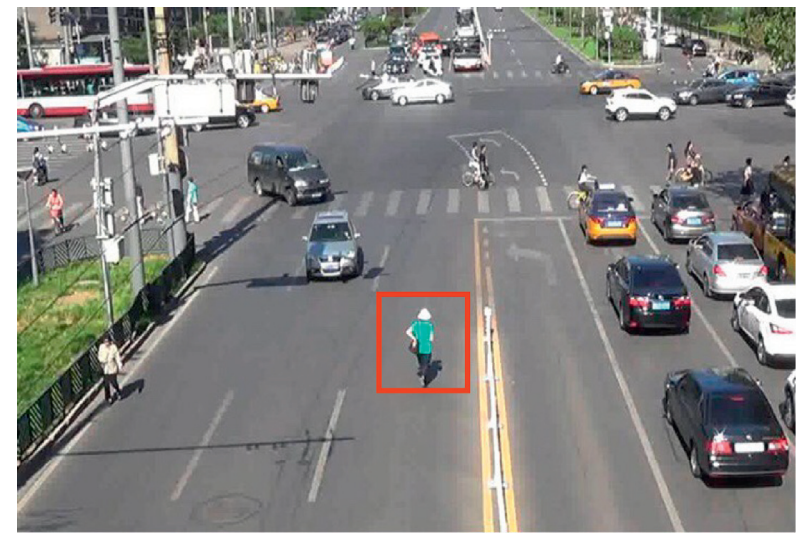

(d)

Figure 1: Violations at signalized intersections under mixed-traffic in Beijing. (a) Running red light at the through phase. (b) Running red light at the left-turn phase. (c) Crossing the intersection diagonally. (d) Intruding into the lane.

of emissions caused by traffic congestion, single point signal timing [8-10], signal coordination between intersections $[2,11,12]$, intersection shape [13], road characteristics [14], and traffic behaviour. And existing research related to intersection emissions can be divided into two categories: (1) intersection emissions estimation and (2) traffic congestion and traffic behaviour at the intersection.

In the existing studies, Rakha et al. [15] studied the impact of acceleration and deceleration behavior at intersections on emissions and found that emission was more sensitive to cruise under the same speed levels. Papson et al. [16] estimate emissions in a combination with the average emission rate at the four driving modes at signalized intersections. However, the aggregation of four driving modes cannot reflect trajectory characteristics. Zhang et al. [17] developed SIDRA model for the emission estimation at the intersection. Gokhale et al. [18] developed a CO concentration model based on the traffic flow pattern at intersections. Braven et al. [19] estimated emissions based on fuel consumption and emission data obtained from an existing emission inventory dataset.

In terms of congestion, Stevanovic et al. [20] suggested that the moderate speed, shortest delays, and fewest stops are the best vehicles' operation of traffic on arterial roads for emissions. Frequent stops and accelerating at intersections can result in high fuel consumption. The accelerations lead to higher fuel consumption rates compared to idling or deceleration [20]. Many researchers studied determining the factors affecting the emission levels at intersections. These studies show that nonsmooth operations and stop-and-go activities are the most important factors for high emissions at urban intersections [21, 22]. More time was spent in acceleration at intersections because of stop-and-go activities. The vehicle's engine power operates at a higher level during acceleration, and it causes excessive emissions $[23,24]$.

In terms of traffic behaviour, Sun et al. [25] quantified the effects of Advanced Traffic Signal Status Warning Systems (ATSSWS) on driving behaviour. The systems can reduce traffic emissions at intersections by reducing unnecessary brakes and accelerations. Przybyla et al. [26] studied the changes of vehicle trajectory and follow-up laws under the distracted driving behaviour. The following model of the front and subsequent vehicles was developed under the distracted behaviour. Mudgal et al. [27] investigated the impact of variability of driving behaviours on vehicle emissions at the roundabout intersection.

However, the quantification of the impact of real-world violations (based on the volumes of vehicle, nonmotor vehicle, and pedestrian) on the operation and emissions was 
not investigated in the existing studies. Therefore, this paper develops a violation blocking model to estimate vehicles' operation and emissions at the protected intersections of arterials and collectors.

\section{Materials and Methods}

This paper includes three steps. (1) The first is determining the arterial and collector intersections as research object by comparing the violations' frequency of pedestrians and nonmotorized vehicles of different types of intersections and signal control. (2) Second is developing the violation blocking model according to the characteristics of the violations. The blocking probability for each vehicle can be estimated based on the volumes of pedestrians and nonmotor vehicles. (3) Third is estimating emissions based on blocking positions and average lost time (see Figure 2) [7].

2.1. Data Source. The paper includes two data sources. (1) Field survey data is used for analysing vehicle behaviors and developing violation mode under the violation at intersections. (2) Emission data is used for quantifying emission.

2.1.1. Field Survey Data. The video data of 5 signalized intersections under mixed-traffic flow were collected in Beijing, China, 2017. The data includes intersection attributes and operation data listed as follows:

\section{(1) Intersection attributes}

(a) Channelization information

(b) Signal information

(2) Operation data

(a) Fundamental data: this includes two parts: (1) the volume of vehicle, nonmotor vehicle, and pedestrian and (2) the time headway of all vehicles with a precision of 0.02

(b) Trajectories data: ten vehicles' trajectories, which are interfered by violations, are collected

(c) Time headway: 388 groups of time headway were collected with a precision of $0.02 \mathrm{~s}$ under the influence of violations

2.1.2. Emission Data. The vehicle emissions data are derived from local emission rates model for light-duty gasoline vehicles $[28,29]$. The emission standard of China III is selected to provide the emission rates for LDVs. The VSP (Vehicle Specific Power) is estimated after the data quality control $[30,31]$.

The emission factors are estimated with the following procedures based on idling time and the number of stops [7]:

(1) Eighty-five VSP distributions are developed according to the number of stops, idling times, and divisions upstream and downstream (see Table 1);

(2) The average emission factors in each VSP bin are estimated and the emission factors in 85 intervals are estimated, which are corresponding to each operation state of intersection vehicles [7]. The emissions can be quantified based on the idling time and number of stops of vehicles before and after violations.

2.2. Determination of Study Object. In Beijing, the arterial roads have abundant traffic volume. Therefore, violation pyramids are developed based on three types of intersections (arterial and arterial, arterial and collector, and arterial and branch) (see Figure 3). Taking pedestrian as an example, the pyramid of violations is divided into three levels: (1) pedestrian volume, (2) violation volume, and (iii) effective violation (interference with vehicles' operation). Effective violation is defined as the violation which can interfere with the vehicles' operation. In the real world, the arterial and collector intersections have a higher proportion of the effective violations, due to the high vehicle volume and limited space (see Figure 3). There are more conflicts and violations between the three components (vehicle, nonmotor vehicle, and pedestrian) in limited space. Therefore, this paper aims to investigate the impact of pedestrian and nonmotorized vehicle violations on emissions at arterial and collector intersections.

At mixed-traffic flow intersection, the category of violations is diverse and their occurrence probability is different. Running red light and crossing the intersection diagonally account for $91.75 \%$ of effective violations. Therefore, the object of this research is running red light and crossing the intersection diagonally at arterial and collector intersections.

\subsection{Impact of Pedestrian and Nonmotorized Vehicle Viola-} tions on Vehicle Trajectories. Figure 4 shows the vehicle trajectories which are affected by pedestrian and nonmotorized vehicle violations in which $x$-axis is time, and $y$-axis is distance. The positive ordinate is the downstream the intersection, and the negative ordinate is the position of queuing vehicles. The red point represents the position and time of the violation. $\tau$ is the sum of driver's reaction time and braking time, and $S_{\tau}$ is the corresponding distance. The dotted lines and solid lines with the same color indicate the same vehicle before and after violations, respectively (see Figure 4).

The vehicle fleet affected by pedestrian and nonmotorized vehicle violations includes two types. (1) The first is idling vehicles (see Figure 4(a)). The trajectory is similar regardless of violations. When idling vehicles delay a startup by violations, the fleet's idling time and the number of stops will increase. (2) Second is running vehicles (see Figure 4(b)). The impact of violations on the trajectories of running vehicles is different. The first affected vehicle has three processes: deceleration, idling, andacceleration. The fluctuation of the fleet is gradually transmitting to the subsequent vehicles and becoming smaller. The following vehicles will have longer idling time such as the fifth car if the fluctuation is small enough. Then, the subsequent vehicles will cross the intersection with the saturated headway. 


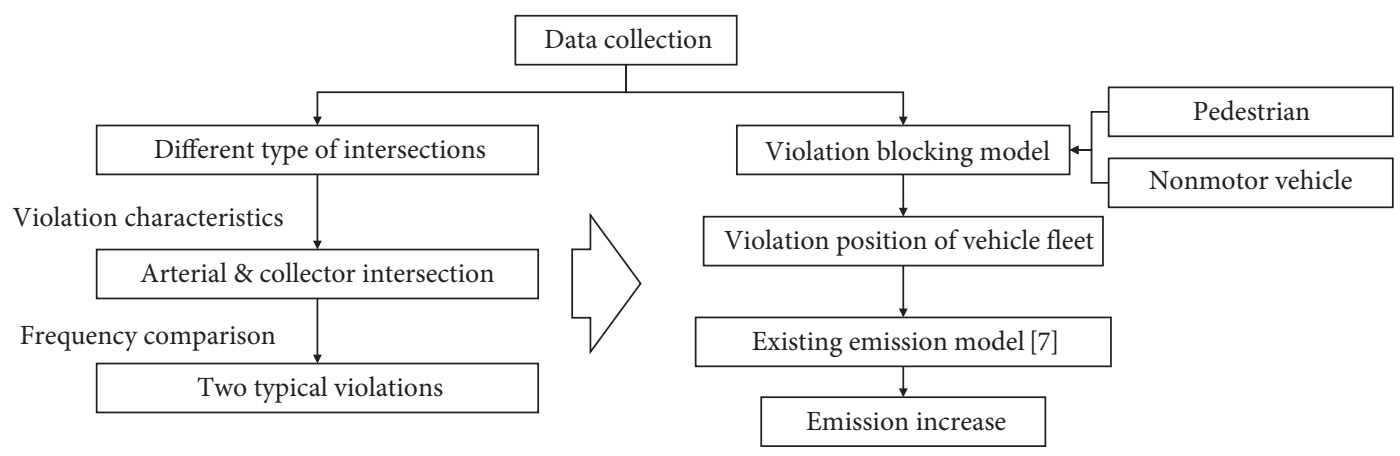

Figure 2: Overview of the methodology.

TABLE 1: Information of the eighty-five VSP distributions [7].

\begin{tabular}{cccc}
\hline The serial number & The spatial position & Number of stops & Idling time(s) \\
\hline 1 & Downstream intersection & 0 & 0 \\
2 & Upstream intersection & 1 & $0-2$ \\
3 & Upstream intersection & 1 & $2-5$ \\
4 & Upstream intersection & 1 & $5-10$ \\
5 & Upstream intersection & & $10-15$ \\
$\vdots$ & & 1 & $135-140$ \\
30 & Upstream intersection & 1 & $140-145$ \\
31 & Upstream intersection & 1 & $145-150$ \\
32 & Upstream intersection & 2 & $10-15$ \\
33 & Upstream intersection & 2 & $15-20$ \\
34 & Upstream intersection & 2 & $20-25$ \\
35 & Upstream intersection & & 2 \\
$\vdots$ & & 2 & $260-265$ \\
83 & Upstream intersection & 2 & $265-270$ \\
85 & Upstream intersection & 2 & $270-275$ \\
\hline
\end{tabular}

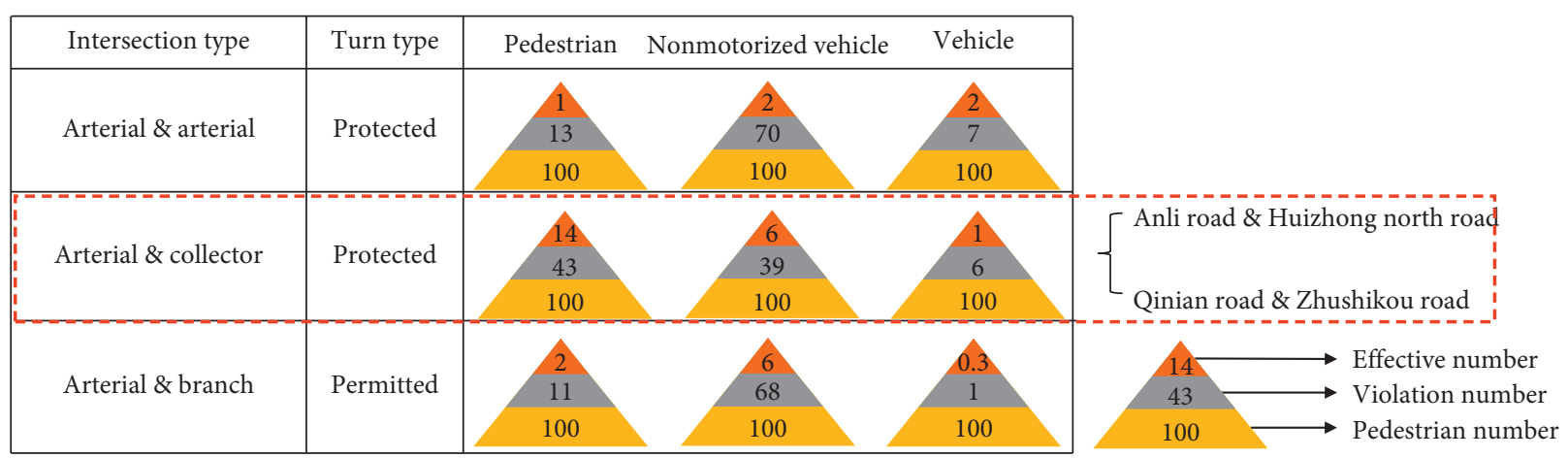

Figure 3: Violation pyramids. Note: protected: go straight and turn left at the same time; permitted: go straight and turn left at the different time.

2.4. Violation Blocking Model. In order to estimate the blocking probability for each vehicle, a violation blocking model is developed based on the volumes of vehicles, nonmotor vehicles, and pedestrians. It is a basis for the estimation of the emission increase of violations.

At the intersection of arterial and collector protected, straight and left-turn vehicles cross the intersection at different time and have different violation characteristics. Therefore, this section includes two scenarios: (1) through phase and (2) left-turn phase. All the violation blocking models are developed based on the 55-cycle data of arterial and collector intersections (Anli and Huizhong North have 25 cycles, and Qinian and Zhushikou have 30 cycles).

2.4.1. Through Phase. Running red light is the main violation of through phase, because the diagonally crossing nonmotor vehicles can cross the intersection twice legally. The $95.6 \%$ of red light runners will lead the head vehicle to 


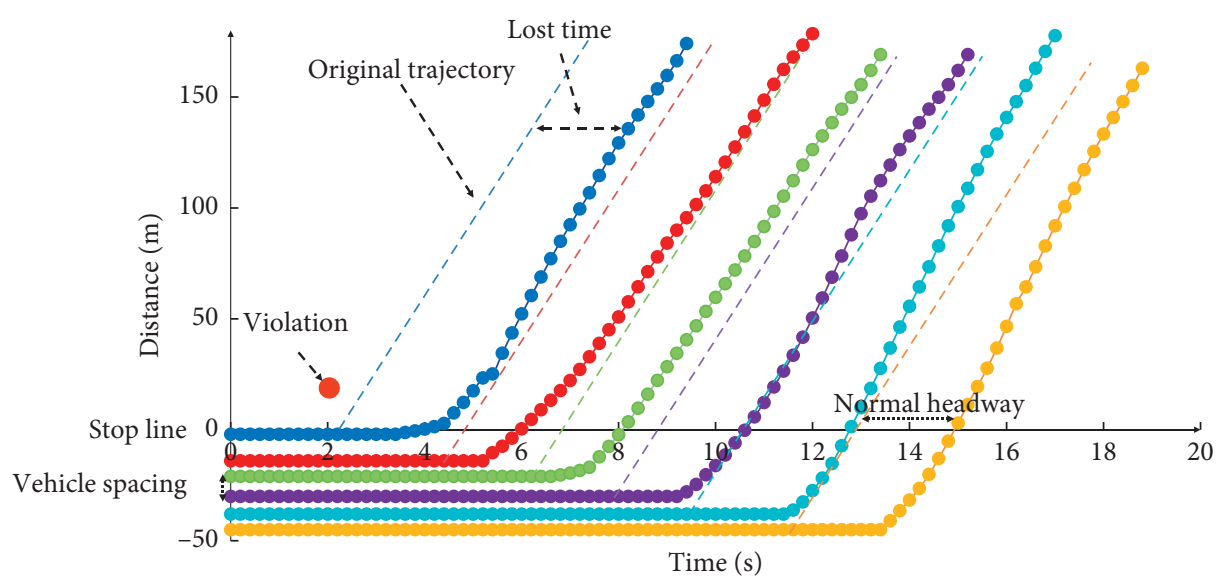

(a)

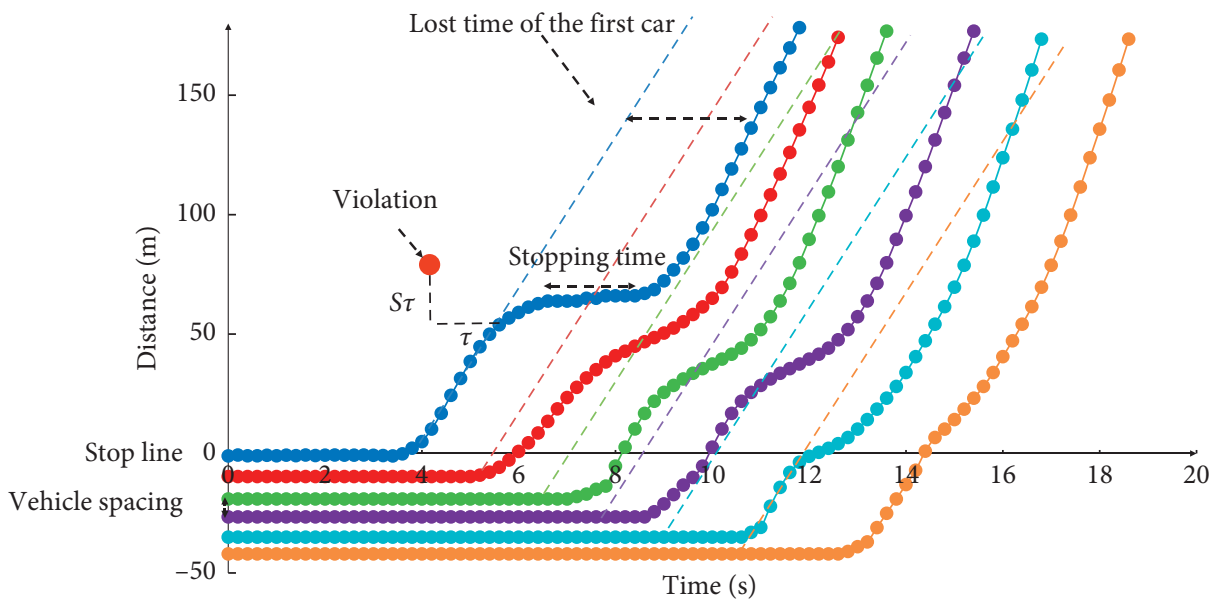

(b)

FIGURE 4: Vehicle trajectories affected by the violation. (a) Idling vehicles. (b) Running vehicles.

delay a start-up or restart; thus, the blocking position can be regarded as the head vehicle at the through phase.

Huang et al. [32] have developed a survival curve to describe the relationship between the probability of running red light and the waiting time.

The blocking probability at the through phase can be estimated based on the probability of running red light and the probability of the fleet being blocked under the running red light condition.

The violation blocking model at the through phase includes three parts: (1) correction coefficient is defined as the probability of vehicle fleet being affected when pedestrian and nonmotorized vehicle violations occur; $k$ equals 0.402 according to the collected data; (2) the volume of pedestrians and nonmotor vehicles is used to estimate how many pedestrians arrive at the waiting area during the specific time; (3) pedestrians and nonmotor vehicles' survival curve is used to estimate how many pedestrians will run red light, and the violation blocking model at the through phase is developed as follows:

$$
\begin{aligned}
P_{\text {through }} & =1-\overline{P_{\text {ped }}} \cdot \overline{P_{\text {non }}}, \\
P_{\text {ped }} & =\sum_{i=1}^{\theta} k \cdot C_{\theta}^{i} \cdot a_{\mathrm{ped}}^{i} \cdot\left(1-a_{\mathrm{ped}}^{i}\right)^{\theta-i} \cdot\left[1-\left(1-\beta_{\mathrm{ped}}\right)^{i}\right], \\
P_{\mathrm{non}} & =\sum_{i=1}^{\theta} k \cdot C_{\theta}^{i} \cdot a_{\mathrm{non}}^{i} \cdot\left(1-a_{\mathrm{non}}^{i}\right)^{\theta-i} \cdot\left[1-\left(1-\beta_{\mathrm{non}}\right)^{i}\right], \\
a_{\mathrm{ped}} & =\frac{V_{\mathrm{ped}}}{C}, \\
a_{\mathrm{non}} & =\frac{V_{\mathrm{non}}}{C},
\end{aligned}
$$

where $P_{\text {through }}$ is the probability that the vehicle will be affected in the through phase. $P_{\text {ped }}$ and $P_{\text {non }}$ are the violation probability of pedestrian and nonmotor vehicles. $K$ is correction coefficient, defined as the affected probability of 
vehicle fleet when violations occur; $k=0.402 . \theta(s)$ is the effective interval of nonmotor vehicle and pedestrian; effective interval is the crossing time of pedestrian and nonmotor vehicle. $I$ is used to describe that there are $i$ pedestrians running red light within the time $\theta . a_{\text {ped }}$ and $a_{\text {non }}$ are the probability of reaching pedestrians per second. $\beta_{\text {ped }}$ and $\beta_{\text {non }}$ are the probability of running red light when pedestrians and nonmotor vehicles arrive. $V_{\text {ped }}$ and $V_{\text {non }}$ (veh) are the volumes of pedestrian and nonmotor vehicle in every signal period. $C(s)$ is signal cycle time.

The two intersections' relative errors are $4.1 \%$ and $8.4 \%$, respectively, after inputting the actual data into the model. The result is acceptable due to the randomness of violation behaviors (see Table 2).

2.4.2. Left-Turn Phase. At the left-turn phase, the vehicle fleet can be blocked anywhere because the runners can insert the fleet easily due to the lower speed and fewer lanes. The violation blocking model at the left-turn phase includes two scenarios: (1) the first vehicle affected by crossing the intersection diagonally and (2) the subsequent vehicles affected by running red light.

Both pedestrians and nonmotor vehicles have violation behaviors. However, few pedestrians will have effective violations which can interfere with vehicle fleet at the left-turn phase, due to the poor mobility of pedestrians and the long distance between pedestrians and vehicles. Thus, the nonmotorized vehicle is the main research object at the left-turn phase.
(1) Violation Blocking Model for Crossing the Intersection Diagonally. The violation blocking model for crossing the intersection diagonally has remarkable scale effect on vehicle fleet. The head vehicle will delay a start-up or restart when several nonmotor vehicles cross the intersection diagonally together.

Based on the above analysis, the model for crossing intersection diagonally includes two steps: (1) the estimation of violation probability based on the volumes of nonmotor vehicles and (2) the estimation of probability that the volume of nonmotor vehicle crossing intersection diagonally exceeds specific scale.

The violation blocking model for crossing the intersection diagonally is fitted by the logarithm model based on the volumes of nonmotor vehicles, and the $R^{2}$ equals 0.826 .

$$
\begin{aligned}
& P\left(N_{\text {non }}\right)=0.1641 \cdot \ln \left(N_{\text {non }}\right)-0.0978 \quad N_{\text {non }} \geq 0, \\
& P\left(N_{\text {non }}\right) \leq 1,
\end{aligned}
$$

where $P\left(N_{\text {non }}\right)$ is the probability of crossing intersection diagonally. $N_{\text {non }}$ is the number of nonmotor vehicles that want to arrive the diagonally opposite of intersections when the turn-left light is on.

The nonmotor vehicles will affect the start of the leftturning fleet, when the number of nonmotor vehicles crossing the intersection diagonally reaches a specific scale. The probability model of the number of violations exceeds a specific scale that can be described as follows:

$$
P_{\text {left,diagonally }}\left(N_{\text {non }}\right)=1-\sum_{i=0}^{N_{\text {scale }}-1} C_{N_{\text {non }}}^{i} P\left(N_{\text {non }}\right)^{i} \cdot\left[1-P\left(N_{\text {non }}\right)\right]^{N_{\text {non }}-i} \text {, }
$$

where $P_{\text {left,diagonally }}$ is the probability of the head vehicle affected. $N_{\text {scale }}$ is the critical scale of violations, $N_{\text {scale }}=6$.

The two intersections' relative errors are $0.32 \%$ and $19.56 \%$, respectively, after inputting the actual data into the model (see Table 3).

(2) Violation Blocking Model for Running Red Light. Nonmotor vehicles, which run the red light during the all-red time, are always besides the left-turn fleet when the left-turn light turns green. And red light runners will only affect the subsequent vehicles due to the existence of the left-turn waiting zone. The existing study indicates that the blocking probability is increasing with time headway [33] (see Table 4). The violation blocking model for running red light at the left-turn phase includes two steps: (1) developing the Gauss distribution of the time headway and (2) estimating the corresponding blocking probability under the specific time headway.

The probability model of time headway is fitted by Gauss model, and $R^{2}$ equals 0.782 . Then, the corresponding blocking probability can be estimated under the specific time headway.

$$
\begin{aligned}
P(t) & =0.0077+0.0521 \cdot e^{-2.3222 \cdot(t-2.2864)^{2}}, \\
P_{\text {left,run }}(t) & =t \longrightarrow G(t),
\end{aligned}
$$

where $P(t)$ means that the probability of the time headway is $t . T(s)$ is time headway. $P_{\text {left, run }}(t)$ is the blocking probability of running red light. $G(t)$ is the correspondence between the time headway and the blocking probability.

2.4.3. Summary of Violation Blocking Model. The model's purpose is to estimate the blocking probability for each vehicle based on the volumes of nonmotor vehicles and pedestrians. The violation blocking model includes two scenarios: through phase and left-turn phase.

At the through phase, the main pedestrian and nonmotorized vehicle violations are running red light, which will lead the head vehicle to delay a start-up or restart. The violation blocking model at through phase includes two input parameters: (1) volumes of pedestrian and nonmotor vehicle and (2) the survival curve which can quantify the relationship between the probability of running red light and the waiting time. 
TABLE 2: Running red light at the through phase: model error.

\begin{tabular}{lccc}
\hline Intersection name & \multicolumn{2}{c}{ Blocking probability } & \\
& Actual blocking (\%) & Violation blocking model (\%) & Absolute error (\%) \\
\hline Anli and Huizhong North & 53.3 & 57.4 & 4.1 \\
Qinian and Zhushikou & 60.6 & 69.0 & 8.4 \\
\hline
\end{tabular}

TABLE 3: Cross intersection diagonally at the left-turn phase: model error.

\begin{tabular}{lccc}
\hline Intersection name & \multicolumn{2}{c}{ Blocking probability } & Absolute error (\%) \\
& Actual blocking (\%) & Violation blocking model (\%) & 12.32 \\
\hline Anli and Huizhong North & 12.00 & 65.23 & 0.32 \\
Qinian and Zhushikou & 46.67 & 19.56 & . \\
\hline
\end{tabular}

TABLE 4: Gap accepted by nonmotor vehicles [33].

\begin{tabular}{lccc}
\hline $\begin{array}{l}\text { Time } \\
\text { headway(s) }\end{array}$ & Refuse block & Accept block & Accept proportion (\%) \\
\hline $1.5-2.0$ & 41 & 0 & 0 \\
$2.0-2.5$ & 163 & 16 & 9 \\
$2.5-3.0$ & 111 & 37 & 25 \\
$3.0-3.5$ & 92 & 48 & 34 \\
$3.5-4.0$ & 57 & 82 & 59 \\
$4.0-4.5$ & 33 & 90 & 73 \\
$4.5-5.0$ & 10 & 56 & 85 \\
$5.0-5.5$ & 8 & 96 & 92 \\
$5.5-6.0$ & 0 & 61 & 100 \\
\hline
\end{tabular}

At the left-turn phase, crossing the intersection diagonally affects the head vehicle's operation, and running the red light affects the subsequent vehicles' blockage. The violation blocking model at left-turn phase includes three input parameters: (1) volumes of pedestrian and nonmotor vehicle, (2) the probability model of running diagonally, and (3) the correspondence between the time headway and the blocking probability.

2.5. Emission Model for Violation Blocking. The existing emission model can estimate the emissions based on the blocking positions. It includes two parts [7]. (1) The linear emission model is developed considering the number of stops and idling times, which is used for estimating emissions under nonviolation and violation conditions. (2) Violation emission model is developed clearly at two levels: trajectory level and traffic flow level (see Figure 5).

At the trajectory level, the study focuses on the first four vehicles affected by pedestrian and nonmotorized vehicle violations. First, the trajectory model of the head car is developed. Then, the Gipps' car-following model is also applied for other three vehicles. Figure 5(a) shows the trajectories under the impact of pedestrian and nonmotorized vehicle violations in which $x$-axis is time, and $y$-axis is distance. The red point represents the location and time of the violation. $\tau$ is equal to the sum of driver's reaction time and braking time, and $S_{\tau}$ is the corresponding distance. The first affected vehicle has three processes: deceleration, idling, and acceleration. The fluctuation of the fleet is gradually transmitting to the subsequent vehicles and becomes smaller. When the fluctuation is small enough, the following vehicles will have longer idling time such as the fifth car. Then, the subsequent vehicles will cross the intersection with the saturated headway. The emissions can be estimated based on the speed and acceleration at 1 second interval (see Figure 5(a)).

At the traffic flow level, the study focuses on the subsequent vehicles after the first four vehicles, and the study is divided into unsaturated and saturated scenarios. It is assumed that the total lost time of the first four effected vehicles is $4 \mathrm{~s}$. In the unsaturated scenario, the subsequent vehicles' idling time increases by $4 \mathrm{~s}$, and the number of stops is still one. In the saturated scenario, two vehicles will transform from one stop to two stops due to the increase of idling time. And the two-stop vehicles' idling time increases by $4 \mathrm{~s}$. The emissions can be estimated according to the developed linear emission model, whose input parameters are the number of stops and idling times (see Figure 5(b)).

The sum of the emissions on these two levels is the total emissions at intersections affected by pedestrian and nonmotorized vehicle violations, which can be estimated as in the following equation [7]:

$$
\mathrm{AE}=\frac{\left(\sum_{x=e}^{e+3} \mathrm{EF}_{\text {increased,trajectory }, x}+\sum_{y=e+4}^{n} \mathrm{EF}_{\text {increased,flow }, y}\right) \cdot D}{\sum_{i=1}^{n}\left(\mathrm{EF}_{\text {upstream }, i}+\mathrm{EF}_{\text {downstream }}\right) \cdot D}-1
$$

where $\mathrm{AE}(\%)$ is the increase of emissions. $E$ is the $\mathrm{e}^{\text {th }}$ vehicle, which is the location of the first affected vehicle. $N$ is the vehicle number of the cycles. $\mathrm{EF}_{\text {increased,trajectory }, x}(\mathrm{~g} / \mathrm{km})$ is the increased emission factors of the first four affected vehicles. $\mathrm{EF}_{\text {increased,flow, } y}(\mathrm{~g} / \mathrm{km})$ is the increased emission factors of the subsequent vehicles after the first four affected vehicles. $\mathrm{EF}_{i}(\mathrm{~g} / \mathrm{km})$ is the normal emission factors of vehicles. $\mathrm{EF}_{\text {upstream }}$ and $\mathrm{EF}_{\text {downstream }}(\mathrm{g} / \mathrm{km})$ are the emission factors at upstream and downstream the intersection. $D$ $(\mathrm{km})$ is the distance of the study range, which is $0.2 \mathrm{~km}$.

\section{Case Study}

Numerical simulations are designed in the case. Based on the violation blocking model and the emission model, the impact of pedestrian and nonmotorized vehicle violations on intersection emissions can be quantified based on the 


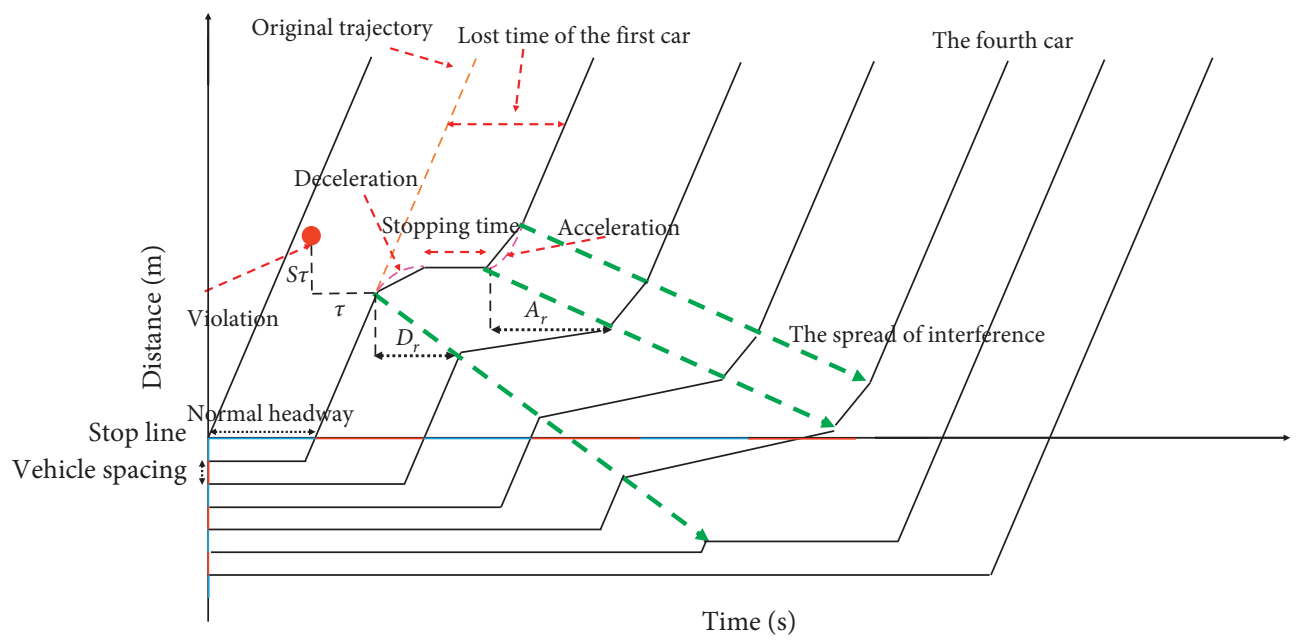

(a)

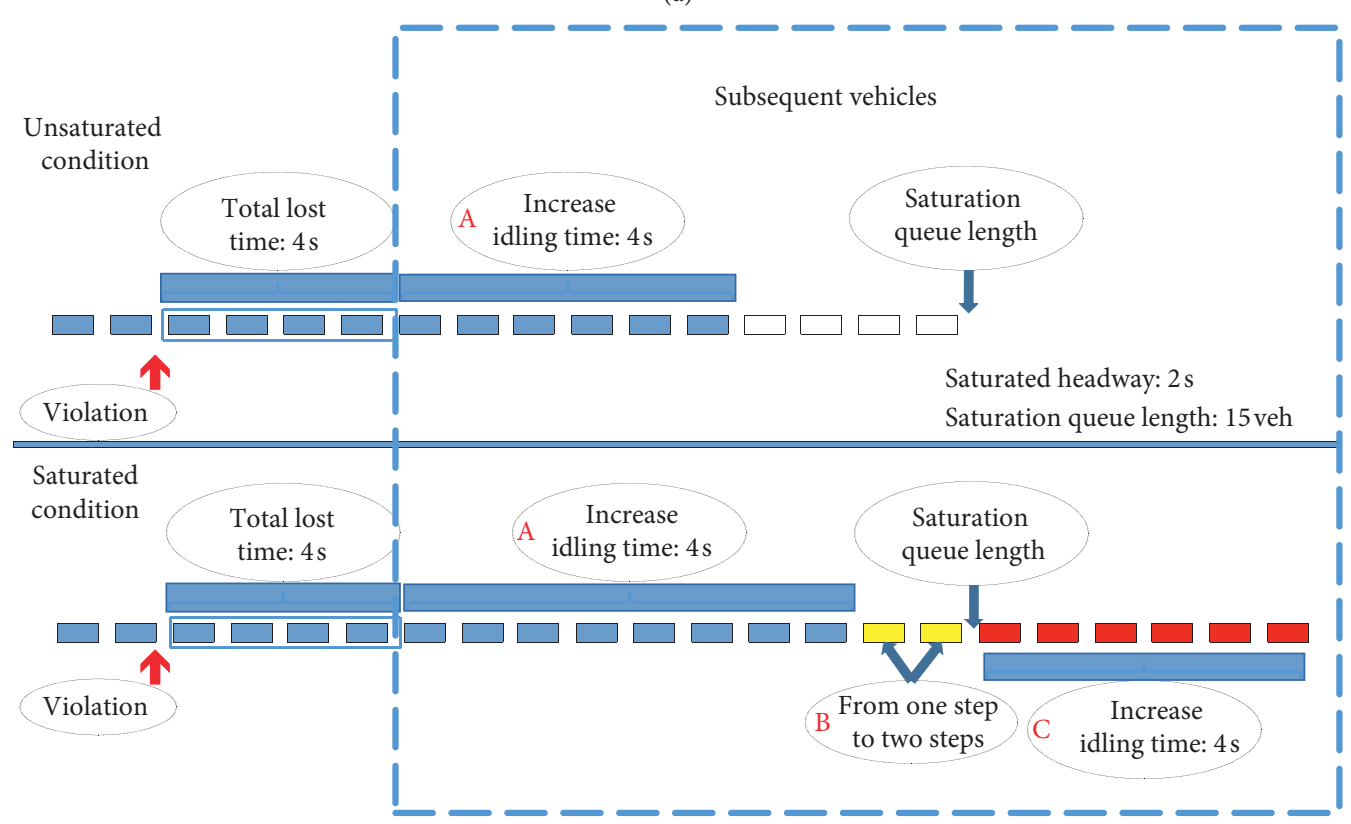

(b)

Figure 5: Vehicle characteristics affected by pedestrian and nonmotorized vehicle violations. (a) Trajectory level. (b) Traffic flow level. [7].

volumes of vehicles, nonmotorized vehicles, and pedestrians. The numerical simulation object of the case is the arterial (north-south) direction of the intersection (see Table 5). The five simulation conditions are listed as follows:

(1) Violation average lost time is $5.52 \mathrm{~s}$

(2) The research range is 200 meters around the intersection

(3) The subject of the simulation is all the vehicles arriving at the research scope

(4) The number of pedestrians and nonmotor vehicles is assumed to be the same

(5) The max number of stops is two times

Repetitive simulations are really necessary due to the randomness of violations. The average increase of 50 groups simulations can improve the simulation accuracy.

\section{Results and Discussion}

The emission increase of pedestrian and nonmotorized vehicle violations has $x$-axis indicating the pedestrian/ nonmotor vehicles volume and $y$-axis indicating the vehicle volume (see Figure 6).

Emissions increase with the vehicle volume approaching the saturated flow rate and the volumes of nonmotor vehicles and pedestrians increasing. The maximum emission increase of $\mathrm{CO}$ for through phase and left-turn phase can reach $16.7 \%$ and $36.4 \%$, as shown in Table 6 .

The emission increase of left-turn phase is higher than that of the through phase, because the blockings have a greater impact on the left-turn phase due to the shorter green time.

As the volume of pedestrians and nonmotor vehicles' increasing, the emission increase has two processes with the 
TABLE 5: Signal and channelization information of case intersection [7].

\begin{tabular}{|c|c|c|c|c|c|c|c|}
\hline \multicolumn{4}{|c|}{ Signal information } & \multicolumn{4}{|c|}{ Channelization information } \\
\hline \multirow{2}{*}{$\begin{array}{l}\text { Phase } \\
\text { North-south straight }\end{array}$} & \multirow{2}{*}{$\begin{array}{c}\text { Green } \\
74\end{array}$} & \multirow{2}{*}{$\begin{array}{c}\text { Yellow } \\
3\end{array}$} & \multirow{2}{*}{$\begin{array}{c}\text { All red } \\
2\end{array}$} & \multirow{2}{*}{ Direction } & \multicolumn{3}{|c|}{ Lane number } \\
\hline & & & & & Straight & Left & Right \\
\hline North-south left-turn & 42 & 3 & 2 & North & 3 & 1 & 1 \\
\hline East phase & 34 & 3 & 2 & South & 3 & 1 & Straight-right \\
\hline West phase & 28 & 3 & 2 & East & 0 & 1 & Straight-right \\
\hline Cycle time & & 198 & & West & 1 & 1 & Straight-right \\
\hline
\end{tabular}

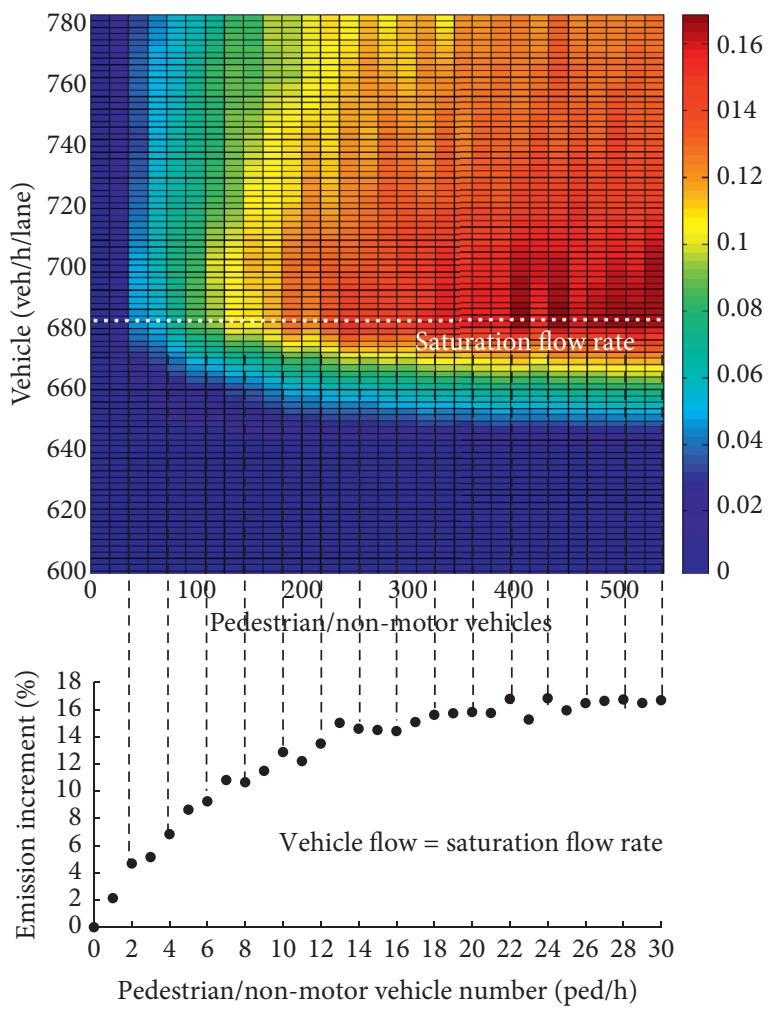

(a)

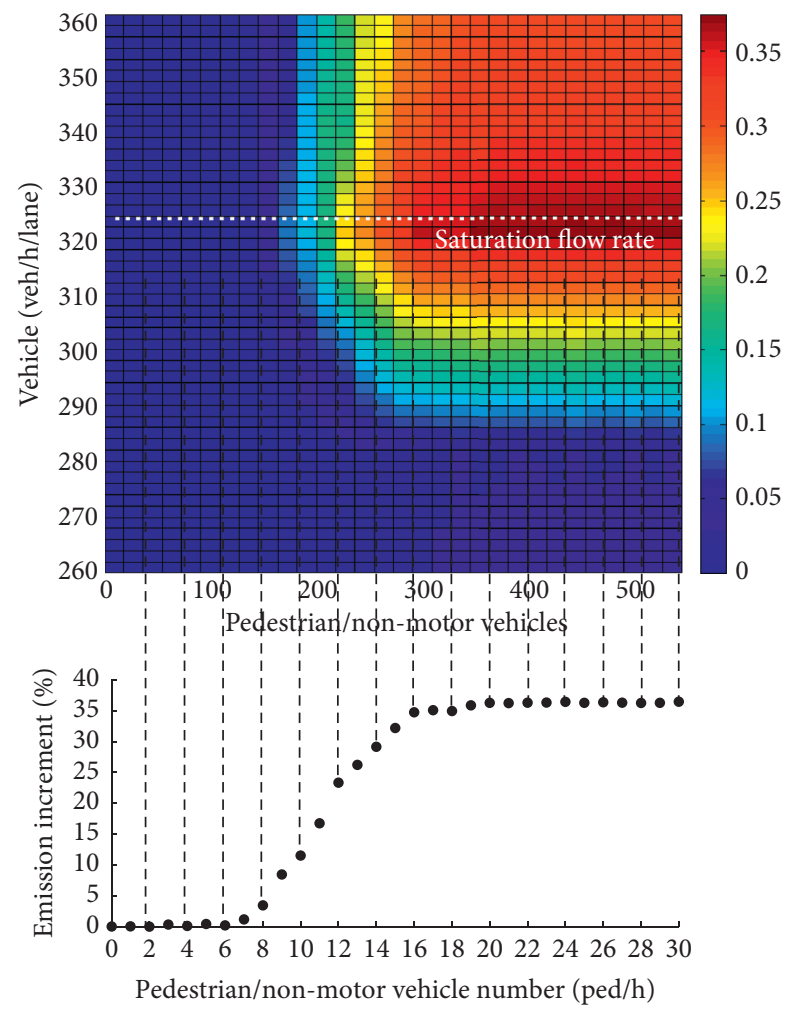

(b)

Figure 6: Emission increase of CO after pedestrian and nonmotorized vehicle violations based on the volumes of vehicles, nonmotor vehicles, and pedestrians. (a) Through phase. (b) Left-turn phase.

TABLE 6: Maximum emission increase of pedestrian and nonmotorized vehicle violations.

\begin{tabular}{lcc}
\hline Pollutant & Through phase (\%) & Left-turn phase (\%) \\
\hline $\mathrm{CO}_{2}$ & 32.6 & 66.6 \\
$\mathrm{CO}$ & 16.7 & 36.4 \\
$\mathrm{NO}_{\mathrm{X}}$ & 20.1 & 47.5 \\
$\mathrm{HC}$ & 28.1 & 59.2 \\
\hline
\end{tabular}

specific number of vehicles: (1) rising and (2) stable. The violation probability is relatively stable when pedestrians and nonmotor vehicles' volume are at a high level.

As the volume of motor vehicles' increasing, there are three periods of the emission increase. (1) The first is rising slowly. Before the traffic flow reaches the saturated flow rate, there is only one stop regardless of the violation; thus, pedestrian and nonmotorized vehicle violations will only increase the idling time. (2) Second is rising rapidly. When the vehicle approaches the saturated flow rate, vehicles transform from one stop to two stops due to pedestrian and nonmotorized vehicle violations. (3) Third is declining steadily. The emission increase will decline steadily when the volume of vehicles exceeds the saturation flow rate. The proportion of the vehicles, which transform from one stop to two stops because of pedestrian and nonmotorized vehicle violations, reduces. The proportion of the vehicles, which always stops two times regardless of violation, is increasing.

\section{Conclusions}

This paper studies the impact of pedestrian and nonmotorized vehicle violations on emissions at arterial and collector protected intersections in the real world. First, the 
characteristics of violations are compared under different types of intersections and violations. Second, violation blocking model is developed to evaluate the blocking position based on the volumes of vehicles, nonmotor vehicles, and pedestrians. Finally, a numerical simulation is conducted to evaluate the impact of pedestrian and nonmotorized vehicle violations on the emissions based on the existing study. Main conclusions can be summarized as follows:

(i) The arterial and collector intersections have a higher proportion of effective violations due to the high vehicle volume and intersection's limited space. Running red light and crossing intersection diagonally are two typical violations at the arterial and collector intersection, which occupy $91.75 \%$ of effective violations.

(ii) The proposed violation blocking model based on the volumes of vehicles, nonmotor vehicles, and pedestrians can be used for estimating the blocking probability for each vehicle at the through phase and left-turn phase.

(iii) The variation of emissions is significant based on the volumes of vehicles, nonmotor vehicles, and pedestrians:

(a) Emissions increase with the vehicle volume approaching the saturated flow rate and the volumes of nonmotor vehicles and pedestrians increasing. The maximum emission increase of $\mathrm{CO}$ for through phase and left-turn phase can reach $16.7 \%$ and $36.4 \%$.

(b) As the volume of pedestrians and nonmotor vehicles' increasing, the emission increment has two processes under the specific volume of vehicles: (1) rising and (2) stable. The blocking probability is relatively stable when pedestrians and nonmotor vehicles' volume are at a high level.

(c) Emission increase is increasing as the vehicle volume is approaching the saturated flow rate. Therefore, as the increasing of the vehicle volume, the emission increase has three processes: (1) rising slowly, (2) rising rapidly, and (3) declining steadily.

This paper develops a method to quantify the impact of pedestrian and nonmotorized vehicle violations on emissions at mixed-traffic flow signalized intersections. Further studies would be conducted on the general model, distribution of lost time of different violations, and red light pedestrians at left-turn phase.

\section{Data Availability}

The emission data come from cited literature in [7, 28-31], and violation data come from manual collection of the project.

\section{Conflicts of Interest}

The authors declare that they have no conflicts of interest.

\section{Acknowledgments}

This study was supported by the National Key R\&D Program of China (no. 2018YFB1600700) and the Natural Science Foundation of China (NSFC) (nos. 71871015, 51678045, and 71901018).

\section{References}

[1] Federal Highway Administration, Carbon Monoxide Categorical Hot-Spot Finding, Federal Highway Administration, New York, NY, USA, 2017.

[2] Y. Zhang, X. Chen, X. Zhang, G. Song, Y. Hao, and L. Yu, "Assessing effect of traffic signal control strategies on vehicle emissions," Journal of Transportation Systems Engineering and Information Technology, vol. 9, no. 1, pp. 150-155, 2009.

[3] L. Zhang, Y. Yin, and S. Chen, "Robust signal timing optimization with environmental concerns," Transportation Research Part C: Emerging Technologies, vol. 29, no. 1, pp. 55-71, 2013.

[4] S. Yagar, B. Han, and J. Greenough, "Real-time signal control for mixed traffic and transit based on priority rules," in Proceedings of the Presented at 71th Annual Meeting of the Transportation Research Board, Washington, DC, USA, March 1992.

[5] M. Franklin, X. Yin, R. Urman, R. Lee, S. Fruin, and R. Mcconnell, "Environmental factors affecting stress in children: interrelationships between traffic-related noise, air pollution, and the built environment," in Proceedings of the HEI Annual Conference, Washington, DC, USA, May 2019.

[6] J. Xing, J. Hua, and P. Hao, "Study on pedestrian crossing rate at signal-controlled intersections," Journal of Technology \& Economy in Areas of Communications, vol. 16, no. 5, pp. 14-19, 2014, Chinese article.

[7] J. C. Huang, G. Song, J. Zhang, C. Li, Q. Liu, and L. Yu, "The impact of violations on vehicles on vehicle emissions at signalized intersections," Journal of Advanced Transportation, vol. 202014 pages, 2020.

[8] B. D. Coensel, A. Can, B. Degraeuwe, I. D. Vlieger, and D. Botteldooren, "Effects of traffic signal coordination on noise and air pollutant emissions," Journal of Environmental Modelling \& Software, vol. 35, no. 4, pp. 74-83, 2012.

[9] J. Lai, L. Yu, G. Song, and X. Chen, "Emission characteristics of heavy-duty diesel transit buses at intersections of urban area: case study in beijing," Journal of Aids Care, vol. 26, no. 10, pp. 1242-1248, 2014.

[10] S. Midenet, F. Boillot, and J.-C. Pierrelée, "Signalized intersection with real-time adaptive control: on-field assessment of $\mathrm{CO} 2$ and pollutant emission reduction," Transportation Research Part D: Transport and Environment, vol. 9, no. 1, pp. 29-47, 2004.

[11] F. Tao, Q. Shi, and L. Yu, "Evaluation of effectiveness of coordinated signal control in reducing vehicle emissions during peak hours versus nonpeak hours," Transportation Research Record: Journal of the Transportation Research Board, vol. 2233, no. 2233, pp. 45-52, 2011.

[12] J. Lv and Y. Zhang, "Effect of signal coordination on traffic emission," Transportation Research Part D: Transport and Environment, vol. 17, no. 2, pp. 149-153, 2012.

[13] J. C. Krogscheepers and M. Watters, "Roundabouts along rural arterials in South Africa," in Proceedings of the Preseented at 93th Annual Meeting of the Transportation Research Board, Washington, DC, USA, January 2014. 
[14] S. Pandian, S. Gokhale, and A. K. Ghoshal, "Evaluating effects of traffic and vehicle characteristics on vehicular emissions near traffic intersections," Transportation Research Part D: Transport and Environment, vol. 14, no. 3, pp. 180-196, 2009.

[15] H. Rakha and Y. Ding, "Impact of stops on vehicle fuel consumption and emissions," Journal of Transportation Engineering, vol. 129, no. 1, pp. 23-32, 2003.

[16] A. Papson, S. Hartley, and K. Kuo, "Analysis of emissions at congested and uncongested intersections with motor vehicle emission simulation," Transportation Research Record Journal of the Transportation Research Board, vol. 2012pp. 124-131, Washington, DC, USA, 2010.

[17] Y. Zhang, J. Lv, and W. Wang, "Evaluation of vehicle acceleration models for emission estimation at an intersection," Transportation Research Part D: Transport and Environment, vol. 18, pp. 46-50, 2013.

[18] S. Gokhale and S. Pandian, "A semi-empirical box modeling approach for predicting the carbon monoxide concentrations at an urban traffic intersection," Atmospheric Environment, vol. 41, no. 36, pp. 7940-7950, 2007.

[19] K. Braven, A. Abdel-Rahim, K. Henrickson et al., Modelling Vehicle Fuel Consumption and Emissions at Signalized Intersection, Publication KLK721. National Institute for Advanced Transportation Technology, University of Idaho, Moscow, ID, USA, 2012.

[20] A. Stevanovic, J. Stevanovic, K. Zhang et al., "Optimizing traffic control to reduce fuel consumption and vehicular emissions: integrated approach with VISSIM, CMEM, and VISGAOST. Transportation research record," Journal of the Transportation Research Board, vol. 2128, p. 105, 2009.

[21] K. Chen and L. Yu, "Microscopic traffic-emission simulation and case study for evaluation of traffic control strategies," Journal of Transportation Systems Engineering and Information Technology, vol. 7, no. 1, pp. 93-99, 2007.

[22] H. Rakha and Y. Ding, "Impact of stops on vehicle fuel consumption and emissions," Journal of Transportation Engineering, vol. 129, no. 1, pp. 23-32, 2002.

[23] H. C. Frey, N. M. Rouphail, and H. Zhai, "Speed- and facilityspecific emission estimates for on-road light-duty vehicles on the basis of real-world speed profiles," Transportation Research Record: Journal of the Transportation Research Board, vol. 1987, no. 1, pp. 128-137, 2006.

[24] H. Zhai, H. C. Frey, and N. M. Rouphail, "A vehicle-specific power approach to speed- and facility-specific emissions estimates for diesel transit buses," Environmental Science \& Technology, vol. 42, no. 21, p. 7985, 2008.

[25] X. Sun, X. Chen, Y. Qi et al., "Analyzing the effects of different advanced traffic signal Status warning systems on vehicle emission reductions at signalized intersections," in Proceedings of the Presented at 95th Annual Meeting of the Transportation Research Board, Washington, DC, USA, December 2016.

[26] J. Przybyla, J. Taylor, J. Jupe, and X. Zhou, "Estimating risk effects of driving distraction: a dynamic errorable car-following model," Transportation Research Part C: Emerging Technologies, vol. 50, pp. 117-129, 2015.

[27] A. Mudgal, S. Hallmark, A. Carriquiry, and K. Gkritza, "Driving behavior at a roundabout: a hierarchical bayesian regression analysis," Transportation Research Part D: Transport and Environment, vol. 26, no. 1, pp. 20-26, 2014.

[28] D. Xie, G. Song, J. Guo, J. Sun, and L. Yu, "Development and application of an online dynamic emission model for traffic networks: a case study of Beijing," in Proceedings of the Presented at97th Annual Meeting of the Transportation Research Board, Washington, DC, USA, June 2018.
[29] C. Li, L. Yu, W. He, Y. Cheng, and G. Song, "Development of local emissions rate model for light-duty gasoline vehicles: beijing field data and patterns of emissions rates in EPA simulator," Transportation Research Record: Journal of the Transportation Research Board, vol. 2627, no. 1, pp. 67-76, 2017.

[30] Report of overall architecture design and technical difficult of the distribution regular pattern of traffic emissions, Technical Report for Center for Transportation Sector Energy Reduction and Emissions Mitigation of Beijing, Beijing Jiaotong University, Beijing, China, 2014.

[31] J. Zhang, L. Yu, J. Guo, Y. Cheng, W. He, and G. Song, "Optimized adjustment of speed resolution and time alignment data for improving emissions estimations," Transportation Research Record: Journal of the Transportation Research Board, vol. 2570, no. 1, pp. 77-86, 2016.

[32] Y. L. Huang and S. Ni, "Analysis on violation behavior of nonmotor vehicles at plane intersection," Journal of Transportation Engineering and Information, vol. 15, no. 2, pp. 64-70, 2017, Chinese article.

[33] L. J. Xu and W. Wang, "Analysis of influence of left-turn nonmotors in signalized intersection," China Journal of Highway and Transport, vol. 19, no. 1, pp. 89-92, 2006, Chinese article. 